Revue d'histoire de l'Amérique française

सES REVUE D.HISTOIRE DE L'AMÉRIQUE FRANÇAISE

\title{
Les Forges Saint-Maurice sous le régime militaire (1760-1764)
}

\section{Marcel Trudel}

Volume 5, numéro 2, septembre 1951

URI : https://id.erudit.org/iderudit/801696ar

DOI : https://doi.org/10.7202/801696ar

Aller au sommaire du numéro

Éditeur(s)

Institut d'histoire de l'Amérique française

ISSN

0035-2357 (imprimé)

1492-1383 (numérique)

Découvrir la revue

Citer cet article

Trudel, M. (1951). Les Forges Saint-Maurice sous le régime militaire (1760-1764). Revue d'histoire de l'Amérique française, 5(2), 159-185.

https://doi.org/10.7202/801696ar d'utilisation que vous pouvez consulter en ligne.

https://apropos.erudit.org/fr/usagers/politique-dutilisation/ 


\section{LES FORGES SAINT-MAURICE SOUS LE RÉGIME MILITAIRE*}

$$
(1760-1764)
$$

En accomplissant la conquête du Canada, les Anglais devenaient les acquéreurs d'un pays riche en pêcheries, en fourrures et en bois de toutes sortes, d'un pays déjà bien organisé au point de vue agricole et doté d'un certain nombre de petites industries, mais ce pays ne possédait alors qu'une seule industrie lourde, celle des Forges Saint-Maurice, à sept milles en amont des Trois-Rivières.

Cette industrie lourde était encore toute récente. Ce n'est pas que le fer que l'on trouvait en abondance dans cette région restât ignoré des Français du dix-septième siècle: Talon, Denonville, Champigny avaient fait examiner et projeté d'exploiter des gisements qu'ils considéraient comme précieux, mais on n'avait pas dépassé le stage des projets et "les forgerons, serruriers, armuriers, taillandiers, etc., restaient tributaires des fournisseurs français qui eux-mêmes s'alimentaient en Suède"1 et l'on laissait intacte une source de revenus qui, suivant le mot du Père Charlevoix, pouvait "enrichir une grande ville"'. Ce n'est qu'en 1730 que François Poulin de Francheville obtient, pour vingt ans, le monopole de l'exploitation du fer dans le domaine qui, depuis 1676, était connu sous le nom de Seigneurie Saint-Maurice ${ }^{3}$. Malgré la création d'une association dite Francheville et Cie, puis d'une nouvelle, Cugnet et Cie, les Forges n'avaient encore presque rien donné en 1736: on avait fait des constructions, on avait tracé des chemins pour l'exploitation, mais le rêve de fournir du fer non seulement au pays, mais même à la métropole, paraissait encore irréalisable. L'établis-

* Extrait d'un ouvrage en préparation: Le régime militaire, 1759-1764.

1. Albert Tessier, "Débuts pénibles de l'industrie lourde au Canada", le Cahier des Dix, XII (1947), 53. Notre résumé de la période française des Forges est d'silleurs fondé en grande partie sur l'historique de Mgr Tessier.

2. Benjamin Sulte, Les Forges Saint-Maurice, "Mélanges historiques" no 6, (1920), 39.

3. P.-G. Roy, Inventaire des concessions en fief et seigneurie, fois et hommages et aveux et denombrements, congervés aux Archives de la Province de Québec (6 vol., Besuceville, 1927-1929), II: 115-117. 
sement prend tout de même de l'importance: la Compagnie Cugnet achète la seigneurie, se fait concéder le fief Saint-Etienne pour en extraire du bois à charbon ${ }^{4}$, refait la première forge et en termine une seconde, construit un haut fourneau et "une grande maison de pierre nettement disproportionnée à l'établissement encore très modeste des Forges"5. Tout cela avait englouti des sommes énormes, cependant que la production restait toujours très maigre ${ }^{6}$. Aussi, en 1741, la Compagnie Cugnet se voit-elle acculée à la faillite, elle avait plus de 500,000 livres de dettes ${ }^{7}$.

C'est alors que "le roi s'en mêle", car cette industrie qui avait reçu de l'État de généreuse avances, pour laquelle on avait fait venir de France des ouvriers spécialisés et édifié à grands frais au milieu des bois, ne pouvait plus être abandonnée, surtout si l'on songe qu'on avait à peine entamé les richesses entrevues. La régie d'Etat (ou "par économie", suivant le mot du temps) connut plus de succès: dans la seule année 1747, on produisit le nombre record d'un million de livres de fer ${ }^{8}$; de 1741 à 1748, on sortit des Forges 22,118 boulets et 662 poêles", pour ne mentionner que ces articles, et Bigot affirmait "que de 1741 à 1752, les opérations marquaient un revenant bon de 24,603 livres" en argent ${ }^{10}$. Au fer en barres, aux poêles, aux marmites et aux boulets, on tenta ensuite d'ajouter la fabrication de pièces d'artillerie: François Le Mercier, qu'on avait au préalable envoyé en France pour en étudier à fond la technique, se mit à l'œuvre avec ardeur ${ }^{11}$, mais les troubles de l'Ohio vinrent bientôt l'accaparer et le distraire des Forges ${ }^{12}$. Ses tentatives démontraient quand même que les Forges pouvaient aussi rendre de

4. Concédé à Marie Boucher en 1684 , ce fief venait d'être réuni au Domaine; il fut rattaché à Saint-Maurice pour ne faire avec ce dernier qu'une seule et même seigneurie (Roy, ibid.).

5. Albert Tessier, "Débuts pénibles de l'industrie lourde au Canada", le Cahier des Dix, XII (1947), 63.

6. 14,000 livres de fer en 1738, par exemple, Albert Tessier, "Le roi s'en mêle, 1741-1760", le Cahier des Dix, XIII (1948), 64.

7. Ibid., 73.

8. Ibid., 77.

9. Ibid., 78.

10. Ibid., 82 .

11. En 1751, il fit couler 106 affats, 424 roues de fer, 200 plateformes de campagnes et 12 mortiers (ibid., 79).

12. Documents relatifs à la monnaie, au change et aux finances du Canada sous le régime français choisis et édités avec Commentaires et Introduction par Adam Shortt (2 vol., Ottawa, 1925-1926), II: 896n. 
précieux services en temps de guerre. Malheureusement, toutes sortes de raisons, y compris la guerre de Sept ans (bien qu'il y ait lieu de s'étonner que la guerre n'ait pas, au contraire, donné aux Forges une très forte impulsion), amenèrent bientôt la décadence de l'établissement: Pierre Kalm et l'ingénieur Franquet reconnaissent tous deux, lors de leur visite des Forges ${ }^{13}$, que les ouvriers sont trop difficiles à obtenir, qu'on doit les payer à des prix exorbitants et que les dépenses manquent d'un contrôle rigoureux; Franquet propose même qu'on retourne à l'affermage ${ }^{14}$ : c'était tourner dans un cercle vicieux. Quoi qu'il en soit, "l'histoire française des Forges, plus encore que celle du Canada, se termine sans splendeur pour la mèrepatrie" ${ }^{15}$.

Lorsqu'en septembre 1760, les Anglais mettent la main sur l'industrie des Forges, ils détiennent, malgré sa décadence, une industrie complètement sur pied. Les Forges ont, depuis quelques années, l'aspect d'un village organisé, auquel on accède facilement des Trois-Rivières par un chemin de trois lieues "dessouché et élargi de 10 à 12 pieds" 16 ou par bateau d'où l'on débarque au pied des rapides pour se rendre ensuite aux Forges par une route de dix arpents ${ }^{17}$. Ce village possède une chapelle en bois ronds ${ }^{18}$ où le récollet Hyacinthe Amiot agit à titre d'aumônier ${ }^{19}$, mais ce qui frappe d'abord la vue, c'est la Grand'Maison extravagante, bâtie en pierre solide, au long toit pointu hérissé de cinq têtes de cheminées ${ }^{20}$. Un magasin général, qui avait la réputation d'être bien fourni, servait aux ou-

13. Pierre Kalm, Voyage dans l'Amérique du Nord, "Mémoires de la Société historique de Montréal", septième livraison, 66-68. - Franquet, Voyages et Mémoires sur le Canada (Québec, 1889), 18-22.

14. Franquet, ibid., 22.

15. Albert Tessier, "Débuts pénibles de l'industrie lourde au Canada", le Cahier des Dix, XII (1947), 82.

16. Albert Tessier, ibid., 61.

17. Ibid.

18. "Une église de pièces sur pièces lattée dehors et dedans enduitte de chaux de quarante pieds de long sur trente de large" (Inventaire d'Hertel de Rouville, septembre 1760, communiqué par Mgr Tessier).

19. Le desservant des Forges était entretenu par le roi. (Franquet, Voyages et Mémoires sur le Canada (Québec, 1889), 21.) Le Père Amiot était arrivé aux Forges en mars 1760 et devait y demeurer jusqu'à la fin d'aout 1762. (P. Odoric-M. Jouve, o.f.m. Les Franciscains et le Canada. Aux Trois-Rivieres (Paris, 1934), 299).

20. Albert Tessier, "Le roi s'en mêle", le Cahier des Dix, XIII (1948), 71. 
vriers leurs rations et les provisions les plus variées ${ }^{21}$. Les bâtiments réservés à l'industrie étaient nombreux: la forge haute, bâtie en pierre, avec halle à charbon et magasin; la forge basse, construite de bois, avec aussi sa halle à charbon et son magasin; un martinet ${ }^{22}$ attenant à la forge basse; un haut fourneau avec moulerie et halle à charbon; une boulangerie avec son four; une écurie de 111 pieds de long; une "dépense pour l'avoine" et six hangars" ${ }^{23}$. A l'écart de ces bâtiments, "six maisons de pièces sur pièces" et "dix-sept barraques de pieux servants a loger les ouvriers" ${ }^{24}$ : ces logements, écrit Franquet, sont "plantés ça et là sans aucune symétrie, ni rapport de l'un à l'autre':25. Voilà tout l'établissement des Forges, en 1760, établissement qui n'existe qu'en fonction de l'industrie. La population elle-même n'est qu'une population ouvrière, mobile et instable comme celle de tout centre ouvrier; le nombre d'âmes, en 1760 , est tombé à 72 , dont onze chefs de maison et 18 garçons: aucun de ces hommes n'est "propre a la guerre" et l'officier chargé du recensement a inscrit un zéro au bas des colonnes réservées aux terres concédées et aux animaux ${ }^{26}$. En somme, onze familles dont l'existence est intimement liée à l'exploitation des Forges, et qui sont restées sur place, alors que les journaliers, dont l'engagement était pour ainsi dire saisonnier, étaient retournés dans leurs paroisses, une fois la besogne terminée.

Dans le traité de capitulation de Montréal, on n'avait rien prévu pour ces familles qui dépendaient aussi étroitement d'une industrie d'État et qui se trouvaient là, presque toutes, par autorité du roi. Alors qu'on décida minutieusement du sort des officiers de l'administration et de l'armée, on ne se prononça point sur le personnel des Forges, "commande" au nom du roi ${ }^{27}$; on demanda

21. Voir l'inventaire qu'en donne Estèbe en 1741 (ibid., 70s.).

22. "Une petite forge que l'on apelle martinnet pour reduire le gros fer aux plus petits Echantillons", (Memoire de John Marteilhe en 1764, C.0. 42, I, 1: 161).

23. Inventaire d'Hertel de Rouville, (septembre 1760), 1s.

24. Ibid., 1.

25. Franquet, Voyages et Mémoires sur le Canada (Québec, 1889), 20.

26. "Recensement des habitants de la ville et gouvernement des Trois-Rivières, septembre 1760", Rapport de l'Archiviste de la province de Québec [RAPQ] pour 1946-1947 (Québec, 1947), 52s.

27. C'est-à-dire enrolé par le roi; sur ces familles envoyées de France par l'Etat, voir Benjamin Sulte, Les Forges Saint-Maurice, "Mélanges historiques" no 6 (1920), 63-80; et AC (Archives du Canada), B 21-2: 94. 
seulement que les papiers des Forges, comme ceux des Bureaur de l'Intendance, du Contrôle de la Marine, du Trésor et du Domaine, restent au pouvoir de l'Intendant et ne soient point visités; il fut répondu, avec la réserve de l'article 21, que les papiers concernant l'administration du pays devraient être remis ${ }^{23}$. Les Anglais restaient donc maîtres d'agir à l'égard des Forges, comme ils l'entendaient: ou poursuivre l'exploitation $d u$ fer ou la laisser tomber.

Ralph Burton, premier gouverneur anglais des Trois-Rivières ${ }^{29}$, arrivait dans un Gouvernement qui n'était pas le Pérou: la traite des fourrures était bien déchue de son ampleur d'antan, l'industrie du canot, qui avait rendu célèbre les artisans des Trois-Rivières, fait à peine parler d'elle ${ }^{30}$ sous le régime militaire; la construction de bateaux, qui ne reprend qu'en 1761 , ne semble pas avoir eu grande influence sur l'économie de la région ${ }^{31}$; le Gouvernement ne produisait même pas assez de blé pour se suffire à lui-même et son bois de chêne subissait avec désavantage la concurrence de celui du lac Champlain ${ }^{32}$. Or, même si en principe le salaire du gouverneur et les dépenses générales de l'administration étaient payés par la caisse de l'armée, il était de l'intérêt du gouverneur, officier de l'armée, de trouver le plus de revenus possible pour diminuer tous ces frais, et cela devenait plus urgent encore quand il s'agissait des ouvriers permanents des Forges, à charge à l'État depuis 1741. Aussi le nouveau régime prit-il connaissance de l'état des Forges dès septembre $1760^{33}$. Le magasin de fer et les hangars n'étaient pas tout à fait vides: il s'y trouvait encore 3,100 livres de fer en barres; ici et là sur les terrains, 200 livres de ferraille et 35,000 livres de

28. Documents relatifs à l'Histoire constitutionnelle du Canada 1759-1791. Choisis et édités avec notes par Adam Shortt et Arthur G. Doughty. (Archives publiques, Ottawa, 1921), 19. (Désormais abrégé comme suit: Doc. const.).

29. Burton disait lui-même qu'il n'avait jamais eu l'occasion de se plaindre de la fortune (BM 21669, 129). Et Haldimand disait de Burton: "Si javois le quart de son bien il y à 3 anné que je ne Serois plus en Amérique que par devoir" (BM 21662, 156v.)

30. Burton y fait seulement allusion dans son rapport de 1763: AC, B 21-1: 11 .

31. De mai 1761 à mai 1763 , on construisit 12 bateaux, de 20 à 100 tonneaux (AC, B 21-1: 12). En décembre 1762, Haldimand parle d'un bateau en construction aux Trois-Rivières et de deux dans les côtes (BM 21661, 256v.); en parlant des côtes, il songeait certainement à Yamaska où Amyot, marchand de Québec, s'occupait de const"uction de bateaux (AC, B 68: 69).

32. Doc. const., Rapport de Burton, 1762, 72.

33. Hertel de Rouville, directeur des Forges, procéda à l'inventaire qu'il signa le 8 septembre 1760 avec Landriève. 
fonte; 800 bennes de mine étaient rendues au pied du haut fourneau et 1,200 à la rivière; il restait 500 barriques de charbon de bois et 300 cordes de bois ${ }^{34}$. La réserve de fonte et de mine n'était donc pas à dédaigner, mais celle de bois était absolument nulle ${ }^{35}$. C'étaient là les faibles ressources dont on disposerait, si l'on décidait de reprendre l'exploitation.

Il fut décidé de la reprendre. Dès septembre 1760, le gouverneur général Amherst enjoignait à Burton d'exploiter la fonte qui se trouvait aux Forges ${ }^{36}$ et, le 1er octobre, François Poulin de Courval, inspecteur des Forges, recevait des ordres précis sur la reprise des travaux: exploiter "la fonte qui est déjà tirée des mines", "retenir sur le même pied que ci-devant les ouvriers dont vous trouverez les noms à la suite de la présente", fabriquer le charbon de bois nécessaire et engager pour cela des journaliers; "vous prendrez sur vous, ajoute le secrétaire du gouverneur, le soin de faire graisser \& relever les soufflets des forges. En un mot de faire faire les petites réparations qui sont absolument nécessaires pour mettre les forges en état d'exploiter peu-à-peu la fonte dont il est parlé ci-dessus" ${ }^{37}$. On lui recommande en même temps de tenir "un compte exacte", conseil qui sera bientôt suivi par un contrôle sévère de toutes les dépenses, par des placards défendant aux ouvriers de se servir du charbon de bois pour chauffer leurs maisons ${ }^{38}$, interdisant aux profanes la coupe du bois et la chasse sur les terres de la seigneurie de Saint-Maurice ${ }^{39}$.

Outre l'inspecteur Poulin de Courval et le contremaître Voligny, qui figurera bientôt sur les états de comptes ${ }^{40}$, on a donc sept ouvriers qui apparaîtront d'une façon permanente sur la liste de paie, de par

34. Inventaire d'Hertel de Rouville, 1.

35. D'après un estimé de $1762,33,000$ livres de fonte pouvaient donner du travail pour un mois (AC, B 21-1: 101). Quant au bois, il en fallait en moyenne 12,000 cordes par année.

36. Rapport des Archives du Canada [ RAC ] (1918), app. B, 85.

37. Ibid., $85 \mathrm{~s}$.

38. RAC (1918), app. B, 144 (placard du 29 septembre 1763); le bois de chauffage leur était d'ailleurs fourni par le Gouvernement, ainsi qu'il appert d'après les comptes.

39. RAC (1918), app. B, 125 et 142 (défenses contre la chasse); 115 (contre la coupe du bois).

40. AC, B 21-1: 86, 165; B 21-2: 56. Ce Voligny, fils de l'ancien directeur Hertel de Rouville (Sulte, op. cit., 167) 6́tait "commis" aux Forges sous le régime français (ibid., 112). 
l'ordre d'octobre 1760: Délorme, Robichon, Marchand, Imbleau, Terreau, Michelin et "Belie"; sauf peut-être un ou deux, ce sont des ouvriers que l'administration française avait fait venir de France spécialement pour les Forges ${ }^{41}$. A ces sept ouvriers permanents, on ajoutera quelque temps après deux gougeats (les fils de Marchand et de Robichon) et deux charretiers ${ }^{42}$, ce qui portera à treize, maîtres et ouvriers, le personnel régulier des Forges sous le régime militaire. Enfin, le chapelain (c'est le récollet Amiot) et deux domestiques de Courval sont au nombre des destinataires des vivres que fournit le Gouvernement ${ }^{43}$.

Le personnel régulier est payé au mois, que les Forges marchent ou non ${ }^{44}$, car la fonte ne se fait que de mai à novembre ${ }^{45}$. L'inspecteur Courval reçoit 200 livres par mois ${ }^{46}$; le contremaître, 100 livres; le fondeur, 58 livres $8 \mathrm{sols}$, excepté pendant l'ouvrage où il est payé 22 sols le cent de fonte et 5 livres par poêle ${ }^{47}$; un chauffeur se voit allouer 58 livres 8 sols; un martelleur, 75 livres, le garde-fourneau, 45 livres; un gougeat, 40 livres; un charretier, 42 livres. Ce qui donne une liste de paie permanente de 892 livres 12 sols, chaque mois ${ }^{48}$.

41. Jean-Baptiste Délorme était maître-fondeur depuis au moins 1738 (ibid., 60); Jean-Nicolas Robichon, marteleur, était passé au Canada en 1740 pour les Forges (ibid., 78); Pierre Marchand, marteleur (AC, B 21-1: 101), était arrivé en 1738 comme chauffeur de haut fourneau (Sulte, 67); Luc Imbleau, chauffeur, se trouvait aux Forges en 1752 (ibid., 112); Antoine Terreau, chauffeur, était employé régulièrement aux Forges depuis longtemps (ibid.); Pierre-François Michelin, aussi chauffeur, arrive de France vers 1737 (ibid., 63); enfin, le dernier qu'on nomme Belie et qui figure comme garde-fourneau en 1762 sous le nom de Bella (AC, B 21-1: 101), serait peut-être ce Bélisle engagé en 1732 (Sulte, 129).

42. AC, B 21-2: 119.

43. RAC (1918), app. B., 89: ordre de Bruyère à Courval, le 2 octobre 1760.

44. AC, B 21-2: 119.

45. AC, B 21-2: 15, 21, 94.

46. Rappelons une fois pour toutes que 12 deniers font un sol, vingt sols font une livre (livre française). Sous le régime militaire, six livres font un dollar. Pour trouver la valeur approximative qui correspond au dollar de 1951, nous avons comparé les prix du régime militaire (prix imposés par le régime pour empêcher la spéculation) et ceux de 1951 et nous avons constaté que la livre française vaut notre dollar. Remarquons enfin que lorsque nous parlons du dollar du régime militaire, nous écrivons dollar, parce que le signe $\$$ n'était pas encore en usage. Nous avons donc le tableau suivant:

12 deniers font un sol; 20 sols font une livre

6 livres font un dollar du régime militaire ou $\$ 6$ (1951).

47. AC, B 21-1: 115 .

48. AC, B 21-1: 101, 115, 119. On déduisait de ces montants les rations fournies par le magasin (AC, B 21-1: 83). Sur une valeur de 4,560 livres de rations fournies, la neuvième partie est en rhum, dans un compte de 1761 (ibid.). 
Ce personnel, évidemment, n'était pas suffisant lorsque les Forges fonctionnaient a pleine capacité; il fallait recourir à des journaliers que l'on payait à la journée ou suivant l'ouvrage exécuté: en temps normal, sous le régime militaire, ces journaliers se trouvaient une quarantaine ${ }^{49}$. Il y avait les "faiseurs de chemins" à 40 livres par mois, les bûcheurs à 30 ou 40 sols la corde, les dresseurs et feuilleurs à 7 sols la corde, les charbonniers qui recevaient 25 sols par corde de bois transformée en charbon ou 50 sols par benne de charbon de bois ${ }^{50}$, les charpentiers à 100 livres par mois, les canoteurs à un écu par jour ${ }^{51}$, des ouvriers à 5 livres par jour, des journaliers à 40 sols $^{52}$. La plupart de ces ouvriers s'occupaient des travaux saisonniers: c'est ainsi que le chemin de la minière étant impraticable l'été, "on ne fait transporter la Mine aux forges que Sur les glaces dans le mois de Janvier, fevrier \& mars par les habitans du Gouvernement des trois rivieres; Ces mêmes habitans viennent la tirer de Son lit dans les mois de Juillet et aoust" "53. Alors que, sous le régime français, ces ouvriers étaient "commandés" au nom du roi et que leurs salaires étaient déterminés d'avance, il paraît, selon un mémoire du régime militaire, que tout se décide désormais de gré à gré et que les journaliers se montrent plus exigeants dans le temps des foins et des récoltes ${ }^{54}$.

Ces quarante journaliers, ajoutés aux treize membres du personnel régulier, ne constituaient certes pas une cité ouvrière bien considérable; ils ne formaient en somme que le tiers de la population ouvrière de $1754^{55}$, mais si l'on songe qu'à la suite de la conquête, l'économie canadienne se trouvait pratiquement paralysée et que les revenus se faisaient extrêmement rares, la reprise de l'exploita-

49. AC, B 21-2: 94.

50. Pour faire marcher le haut fourneau et les forges, il fallait normalement 10,000 cordes de bois produisant 3,500 bennes de charbon (ibid., 95). Sulte, dans son Histoire des Forges, p. 102, décrit la fabrication du charbon de bois.

51. L'écu valait d'abord 6 livres (RAC (1918), app. B, 3s), puis par une ordonnance de Haldimand, il fut porté à 6 livres 12 sols, le 7 aout 1762 (ibid., 134).

52. Pour tout ce tableau, voir AC, B 21-1: 86, 87, 115; B 21-2: 94.

53. C.0. 42, I-1: 161 .

54. AC, B 21-2: 94. Ce mémoire, daté de juin 1765, décrit l'exploitation des Forges telle qu'elle se faisait sous le régime militaire; juin 1765 marque la date de la liquidation des comptes de l'administration militaire.

55. Selon Sulte, il y avait sur la liste de paie de 1754, 20 chefs de famille et 132 personnes (Les Forges Saint-Maurice, 121). 
tion des Forges dut sembler une opération d'envergure et très profitable pour la population du Gouvernement des Trois-Rivières.

Pour se conformer aux instructions d'Amherst, on se contenta dans le début, de réduire en barres "la vieille fonte répandue autour des forges"56. Lorsque l'opération se trouva terminée en juin 1761, cette vieille fonte avait donné 30,061 livres de fer qui, ajoutées aux 3,100 livres laissées par l'administration française, formaient un total de 33,161 livres de fer ${ }^{57}$. Pendant cette période de neuf mois (au cours de laquelle le travail n'avait pas été continu), on avait da payer en salaires ou en rations 7,626 livres 8 sols $^{58}$; la coupe et le transport de 350 cordes de bois, le foin et l'avoine de sept chevaux et quelques autres dépenses avaient fait monter les frais d'exploitation, soustraction faite des rations fournies, à 6,629 livres 2 sols, soit une valeur de 1,104 dollars $83 / 100$, cours du régime militaire ${ }^{59}$. En évaluant le fer à 60 dollars le millier, ainsi que le fait habituellement le gouverneur, on obtient une valeur de 1,989 dollars 66/100 et, par conséquent, un surplus de 884 dollars $83 / 100$ pour cette première période de neuf mois ${ }^{60}$. Burton, heureusement, n'avait pas eu à soutenir la dépense très coûteuse d'aller extraire la mine, il n'avait eu qu'à transformer en fer ce qui se trouvait déjà rendu à la porte du haut fourneau. Il restait quand même évident qu'une sage administration pouvait tirer de bons revenus des Forges, si on leur faisait donner leur plein rendement.

C'est pourquoi, Burton décida non seulement de poursuivre l'exploitation telle qu'on l'avait commencée, mais même de reconstruire les fourneaux. C'était une dépense assez lourde pour une entreprise considérée comme temporaire. Ce travail dura près d'un mois: il fallut pour cela se rendre à une carrière de pierre calcaire, située sur la rive opposée du Saint-Maurice, y tailler 72 pieds carrés de pierre, la transporter sur des embarcations en haut des rapides et la voiturer jusqu'aux fourneaux. Lorsque la construction se trouva terminée, on avait dépensé 1,056 livres $^{61}$.

56. AC, B 21-1: 123.

57. Ibid.

58. AC, B 21-1: 83 . Une valeur de $\$ 7,626$. en 1951 . Notons que sur ces 7,626 livres, une valeur de 4,080 livres avait été fournie en rations.

59. AC, B 21-1: 81-83, 92s.

60 . Environ $\$ 5,300$ en 1951 . On vendait le fer a 60 dollars le millier, mais il est arrivé aussi de le vendre à 65 et à 70 dollars (AC, B 21-1: 118).

61. Pour les divers item de ces dépenses, voir AC, B 21-1: 84 . 
Autre contretemps, il n'y avait plus de charbon de bois. Or la fabrication en était bien couteuse. Depuis les trente ans que l'on exploitait les Forges, le bois à charbon se faisait rare dans les environs immédiats de l'établissement, il fallait maintenant aller le chercher bien au delà du Saint-Maurice, dans les profondeurs. On commença donc par établir un chemin solide depuis les Forges jusqu'à la tête des rapides, et là on entreprit d'installer un service de traversier: pour donner une idée des frais que tout cela pouvait entraîner, signalons, en passant, qu'il fallut envoyer trois hommes en canot à Québec pour l'achat d'un câble de 90 toises et que ce câble et ce voyage coûtèrent, à eux seuls, 606 livres $^{62}$. Une fois le service organisé, il fallut ouvrir des chemins dans la forêt pour en sortir le bois, chemins qui, en trois mois, firent débourser 600 livres $^{63}$. Restait le bûchage: on avait besoin de 4,200 cordes de bois. On dut faire appel aux habitants des paroisses en les mobilisant en corvée, ainsi que l'administration les y avait habitués. Burton, le 20 juillet 1761, donna donc ordre aux capitaines de milice d'envoyer des habitants de leurs paroisses "pour bûcher chacun quinze cordes de bois aux forges... Vous leur ordonnerez, précisait la lettre, d'apporter avec eux leurs haches \& des vivres pour le temps qu'ils mettront a bûcher leur bois. Vous les enverrez en droiture à Mr Courval aux forges, de qui ils recevront les ordres. Ce travail presse, donnez vos ordres incessamment \& avertissez les qu'ils seront payés" ${ }^{64}$. On leur donna 40 sols la corde: les conditions étaient donc assez avantageuses ${ }^{65}$. Puis, les 4,200 cordes bûchées, il resta à les transformer en charbon, ce qui se fit à raison de 25 sols la corde ${ }^{66}$. D'autres dépenses vinrent grever lourdement le budget: on eut à travailler au chemin qui conduit des Forges au pied des rapides, où l'on embarquait le fer pour Trois-Rivières; on dut faire venir des charbonniers du Gouvernement de Montréal en leur remboursant les frais de voyage; un "chemin d'eau" s'étant brisé, il fallut envoyer chercher des maçons à Baie-Saint-Antoine pour le réparer; il en coûta 363 livres pour

62. AC, B 21-1: 86. Soit \$606. en 1951.

63. Ibid. L'administration française avait dâ, sans doute, faire de ces chemins pour la sortie du bois, mais il ne semble pas qu'on s'en soit servi, sous le régime militaire, ou bien on les a refaits. Quoi qu'il en soit, les comptes disent "faire des chemins".

64. RAC (1918), app. B, 107.

65. AC, B 21-1: 86. Soit $\$ 2$ d'aujourd'hui, par corde bûchée.

66. Ibid. 
réparer deux forges, 600 livres pour le bois de chauffage fourni aux ouvriers ${ }^{67}, 1,288$ livres pour le foin et l'avoine de six mois, 1000 livres pour diverses fournitures, et ainsi de suite. Bref, de juillet a la fin de décembre 1761 , on avait dépensé 32,952 livres 8 sols 10 deniers, soit 5,492 dollars 4/100 pour le maintien des Forges ${ }^{68}$.

Que retirait-on du travail de cette période? Du produit de la mine, "on fabriqua des barres de différentes grosseurs" et "on fit entrer en magasin" 97,723 livres de fer; on avait fondu, outre cela, 180 "poiles de fer" ${ }^{69}$. En évaluant toujours le millier de fer à 60 dollars et en y ajoutant 18 dollars $1 / 3$ pour chaque poêle ${ }^{70}$, on obtient une valeur globale de 9,163 dollars $38 / 100$. On terminait donc l'année avec un surplus de 3,669 dollars $34 / 100$, malgré les réparations considérables qu'on avait dú faire ${ }^{71}$. Les ouvriers retombent ensuite dans leur chômage saisonnier, en attendant de reprendre la besogne au début de mai 1762 .

Un autre que Burton allait maintenant, au moins d'une façon provisoire, présider à l'exploitation. Le 5 mai, le colonel Frédéric Haldimand, Suisse de langue française ${ }^{72}$, arrivait aux Trois-Rivières pour y remplacer Burton qu'Amherst destinait à l'expédition de la Havane $^{73}$. Burton pouvait partir, satisfait du travail accompli, au moins dans le domaine de l'industrie: sous son administration de 20 mois, les Forges avaient produit 127,784 livres de fer et 180 poelês, soit pour une valeur d'environ $\$ 84,000$ d'aujourd'hui et on avait distribué en salaires plus de $\$ 42,000 \mathrm{du}$ même cours ${ }^{74}$. Aussi ne faut-il pas s'étonner de l'importance qu'il accorde aux Forges Saint-Maurice dans le rapport qu'il rédige sur le Gouvernement des Trois-Rivières, au printemps de 1762, à la demande du gouverneur général Amherst"75. "Apparemment, écrit Burton, les Français n'ont

67. On fournissait aux ouvriers 350 cordes de bois.

68. AC, B 21-1: 85-90. Environ $\$ 32,952$ de 1951 .

69. AC, B 21-1: 123s.; B 21-2: 10 .

70. Le même poêle coûterait aujourd'hui $\$ 110$. Ces poêles étaient des poêles français avec fleurs de lis, parce que le Canada n'appartenait pas encore définitivement à l'Angleterre.

71. Ce surplus équivaut à environ $\$ 22,016.00$ d'aujourd'hui.

72. Haldimand était vraiment de langue française: c'est en français qu'il écrit habituellement à Amherst et à Burton; Amherst lui répond presque toujours en français, et même aussi Burton. Ses papiers personnels sont toujours rédigés en français. Il arrive aux Trois-Rivières le 5 mai (AC, B 21-1: 35).

73. Burton part le 16 mai (ibid.).

74. 42,000 livres ou 7,000 dollars du régime militaire.

75. Doc. const., 67-77. 
retiré aucun avantage de ce gouvernement; ils ont complètement négligé certains moyens de se créer des ressources ou abusé de celles qui auraient pu améliorer leur sort ou dont ils auraient pu tirer des profits. La principale source de revenus dont ils ont abusé est celle provenant des mines de fer et des forges." Le fer tiré du minerai, ajoute-t-il, est d'une qualité "tellement supérieure, que d'après les dernières expériences faites par ordre de Son Excellence le général Amherst, cet article a été trouvé préférable à n'importe quel produit en Amérique, et même à celui importé de la Suède". Le grand nombre d'employés inutiles, les salaires élevés, la cherté des provisions et les fraudes de l'intendant avaient rendu "onéreux" pour la France "cet établissement qui aurait pu lui être profitable"; en faisant les réparations nécessaires et en ajoutant même deux autres forges et un fourneau, "la couronne retirerait de grands avantages à améliorer cette industrie qui fournirait à la marine de Sa Majesté un excellent fer pour la construction de vaisseaux"76. Plus tard, soit au printemps de 1763 , le Board of Trade demanda de nouveaux détails sur l'état du Canada ${ }^{77}$ et, dans la liste des questions auxquelles on priait les gouverneurs de répondre, il y en avait une sur les mines ${ }^{78}$ et une autre sur les forges ${ }^{79}$. Burton répondit à peu près dans les mêmes termes qu'en 1762: il parla de l'abondance des mines de fer dans son Gouvernement, en précisant que seules les mines des Forges Saint-Maurice étaient exploitées; sur les Forges elles-mêmes, il n'ajoute pas grand'chose à ses observations de 1762, sauf qu'il propose qu'on étende le domaine des Forges jusqu'au delà du SaintMaurice, dans la seigneurie du Cap-de-la-Madeleine ${ }^{80}$, afin de procurer à l'établissement plus de bois à charbon, une carrière de pierre calcaire pour la fonte du minerai et pour les réparations, et même des terres à cultiver le foin et l'avoine, ce qui dispenserait d'une dépense considérable pour la nourriture des chevaux; dans ces conditions, conclut-il, on pourrait fournir avec avantage à la marine de Sa Majesté, du fer propre à la construction des navires.

76. Ibid., 71s.

77. Le Board of Trade adressa ces questions aux gouverneurs le 9 mars 1763 et Burton envoya ses réponses dès le 31 mai suivant. On trouvera le texte de ces réponses dans AC, B 21-1: 1-31, B 7: 61-83; C.O. 42, I-1: 34-62.

78. La question 11 (AC, B 21-1: 15).

79. La question 16 (ibid., 22-23).

80. Appartenant aux Jésuites. 
Ces enquêtes du gouvernement anglais ont pu faire supposer que les Forges avaient joué un rôle primordial dans les délibérations du traité et qu'elles avaient amené l'Angleterre à préférer le Canada à la Guadeloupe. Remarquons d'abord que sur la centaine de pamphlets, qui furent publiés de 1759 à 1763 sur les termes du traité, deux seulement, suivant la démonstration qui a été faite, ont pu exercer quelque influence sur le cours des délibérations ${ }^{81}$ : or, de ces deux pamphlets, un seul, qui est de 1760 , parle du fer, et encore parle-t-il très en général du fer que l'on tire des colonies d'Amérique, en mentionnant ce produit en dernier lieu, à la suite des fourrures, des pêcheries, du tabac et de l'indigo ${ }^{82}$. De plus, la même démonstration établit que c'est la France qui, la première, parla, en 1761, de céder le Canada et que Pitt en accepta l'offre immédiatement, au moins en principe ${ }^{83}$ : or, à ce moment, il n'était encore parvenu aucun rapport officiel sur les Forges ${ }^{84}$. De leur côté, lorsque les Chambres de Commerce de l'Ouest de la France, averties de l'offre de Choiseul, protestent contre le projet d'abandonner le Canada à l'Angleterre, elles ne disent pas un mot des Forges ni même du fer, dans l'énumération des produits canadiens: on parle de fourrures, de pêcheries, de bois de construction, de chanvre et même de tabac, mais on semble ignorer qu'il existe au Canada une industrie du fer ${ }^{85}$. Des deux rapports de Burton, l'un rédigé en avril 1762 et l'autre en mai 1763, seul le premier a pu jouer un certain rôle puisque la signature des préliminaires de la paix est du 3 novembre 1762 . De fait, il fut question nommément des Forges Saint-Maurice dans la discussion des articles préliminaires: Shelbourne, alors chargé des négociations par le ministère Bute, qui avait succédé à celui de Pitt, travailla vivement à faire préférer le Canada aux îles à sucre et il cita, entre autres produits, le fer des Forges Saint-Maurice ${ }^{86}$.

81. Marjorie G. Reid, "Pitt's decision to keep Canada in 1761," The Canadian Historical Association, Annual Report 1926, 27ss. Il s'agit des opuscules intitulés $A$ Letter Addressed To Two Great Men, 1760 et Remarks On The Letter Address'd To Two Great Men (c'est la deuxième édition de ce dernier document que nous avons consultée).

82. A Letter Addressed To Two Great Men, ibid., 34.

83. Reid, article cité, 22.

84. Le premier rapport ne parvint en Angleterre qu'au cours de l'été 1762.

85. RAPQ (1924-25), 201-228.

86. R.C. Rowe, article sur les Forges Saint-Maurice, dans le Canadian Geographical Journal, IX (1934), 1: 14-22. 
D'ailleurs, de l'avis même des hommes d'État, il ne pouvait y avoir de paix possible en Amérique aussi longtemps que la France garderait le Canada ${ }^{87}$; on sait également que l'un des grands objectifs de la guerre de Sept ans en Amérique, sinon le plus grand, était la possession de la vallée de l'Ohio et que, de toute façon, c'étaient les fourrures et les pêcheries qui comptaient surtout, pour ne pas dire uniquement. Dans ces conditions, on peut affirmer que le fer canadien n'a pas compté pour un item de première importance dans les richesses que l'on pouvait attendre de la nouvelle conquête. Quoi qu'il en soit des conséquences du rapport Burton, Haldimand se montrera aussi enthousiaste que son prédécesseur et il écrira à Amherst que le fer des Forges est toujours très bon, moins sujet à la rouille que tout autre fer et que si l'amirauté adopte le fer des Trois-Rivières, on pourra en fabriquer n'importe quelle quantite ${ }^{88}$. Il n'y avait pas là vantardise: Kalm, originaire d'un pays grand exportateur de fer, avait retenu, en 1749, la haute qualité du fer, supérieure à celui d'Espagne, plus résistant à la rouille et facile à extraire ${ }^{89}$, et le contrôle rigoureux qui, selon Franquet, manquait à l'administration des Forges $^{90}$, le régime militaire l'appliquait avec vigueur: il y avait profit à exploiter les Forges et le premier terme de Burton le démontrait aisement.

L'exploitation reprit donc en mai 1762 , sous la surveillance méticuleuse du gouverneur intérimaire, Haldimand ${ }^{91}$. Mais les Forges restaient toujours délabrées et Haldimand attendrait "avec plaisir l'ordre de les faire rétablir"92. Cependant, cet ordre ne venait pas, le gouverneur général Amherst n'avait encore reçu aucune réponse de la métropole sur les réparations à entreprendre ${ }^{93}$. Pendant qu'on attendait ainsi la décision des autorités supérieures, l'arbre de la grande roue des forges d'en-bas se rompit de pourriture et l'on dut faire tout l'ouvrage dans les forges d'en haut, ce qui retardait

87. Voir l'article de Reid et les pamphlets cités.

88. Lettre à Amherst, le 25 juillet 1762, BM 21661, 216.

89. Pierre Kalm, Voyage dans l'Amérique du Nord, "Mémoires de la Société historique de Montréal", septième livraison, 67.

90. Franquet, Voyages et Mémoires sur le Canada (Québec, 1889), $21 \mathrm{s.}$

91. Il écrivait a Amherst, le 24 mai 1762: "L'ouvrage des Forges continue a l'Ordinaire et toute la fonte Sera reduitte en barres dans le Millieu de Juillet" (BM 21661, 197).

92. Lettre a Amherst, le 22 juin 1762, BM 21661, 204v.

93. Burton à Haldimand, le 4 juillet 1762, BM 21669, 2. 
l'exploitation ${ }^{94}$. Haldimand envoya donc à Amherst un état du bois de chêne nécessaire à la reconstruction; comme il ne s'en trouvait plus sur les lieux, il proposa d'en faire venir du lac Champlain et Haldimand, économe en tout, fait remarquer qu'en faisant couper par les soldats un peu plus de chênes qu'il n'en faut pour le fort qu'ils sont à construire, on évitera des dépenses extraordinaires ${ }^{95}$. Les ouvriers, qui ne pouvaient s'accorder pour travailler tous dans les mêmes forges, trouvèrent, en attendant, le moyen de réparer les forges d'en bas avec du sapin: avec des précautions, cela pouvait tenir temporairement ${ }^{96}$. Entre temps, Amherst annonçait à Haldimand qu'il avait donné ordre de faire couper du chêne pour réparer les forges $^{97}$.

Les dépenses qu'on entreprend pour réparer solidement les forges, s'expliquent par la nouvelle orientation que l'on donne à cette époque a l'opération de cette industrie. Au cours du printemps, on s'était contenté de mettre en barres "les restes de fer qui Se Sont trouves autour du fourneau"98, mais cette ressource s'épuisant, Haldimand avait proposé à Amherst qu'on entreprit de fondre de vieilles pièces d'artillerie ${ }^{99}$. Amherst accepta volontiers, à condition qu'il y eât profit à le faire: Murray et Gage, respectivement gouverneurs de Québec et de Montréal, feraient le relevé de leur vieux fer et l'on enverrait même aux Forges le fer que l'on avait accumulé en quantité énorme à Halifax ${ }^{1}$. Murray, pour sa part, répondit qu'il pouvait envoyer aux Trois-Rivières 350 tonnes de vieux fer $^{2}$. Haldimand songeait même au vieux fer d'Oswego, de Niagara et du fort Augustus, à condition, bien entendu, qu'on ne se contente pas de réparer les Forges, mais qu'on les rétablisse à neuf ${ }^{3}$. La guerre de Sept ans en Amérique avait enlevé aux Français un butin de guerre énorme et amené en même temps la mise au rancart d'un nombre considérable de pièces d'artillerie démodées ou détériorées; dans

94. Haldimand à Amherst, le 24 juillet 1762, BM 21661, 215.

95. Ibid. le 25 juillet, $1762,216$.

96. Ibid., le 25 aout $1762,223$.

97. Amherst à Haldimand, le 11 aout 1762 ibid., 220v.

98. Haldimand à Amherst, le 24 juillet 1762 , ibid., 215.

99. Amherst à Haldimand, le 24 juillet 1762, ibid., 205-205v. La réponse d'Amherst est ainsi rédigée qu'on ne peut douter que la proposition ne vienne d'Haldimand.

1. Ibid.

2. Haldimand a Amherst, le 25 aout 1762, ibid., 223.

3. Ibid. 
l'esprit d'Haldimand se formait un programme d'envergure: faire des Forges Saint-Maurice un véritable centre industriel qui servît de débouché au vieux fer des forteresses de l'Amérique du Nord.

Malgré leur état délabré, les Forges avaient donné, en 1762, un rendement considérable. Du 10 mai au 31 juillet, on tire de la fonte 82,437 livres de fer; du 23 août au 16 novembre (date ultime de l'opération), les vieilles pièces d'artillerie avaient produit en fer 68,039 livres. Aucun poêle n'est sorti des Forges, cette année-là ${ }^{5}$. La production totale de l'année se chiffrait donc à 150,476 livres, soit un surplus de près de 25,000 livres sur 1761. L'opération de 1762 avait couté 5,956 dollars $83 / 100^{6}$. En évaluant le fer à 60 dollars le millier, on a une valeur globale de 9,028 dollars $56 / 100$, ce qui donne pour 1762 un profit de 3,071 dollars $73 / 100^{7}$.

Nous n'avons là, il est vrai, qu'un profit théorique, puisque le fer avait le temps de se refroidir longuement avant de passer chez les clients, qui n'étaient pas nombreux. Les clients réguliers étaient surtout Jacques Terroux et François Lévesque, marchands de Québec, et Haldimand n'avait pas souvent la chance de vendre le fer en grosse quantité, comme cela s'est produit avec la Bruère de Boucherville qui, en 1762, en acheta pour 37,000 livres pesant ${ }^{8}$. Lorsque Haldimand fit la récapitulation des ventes, il trouva de vendu 55,143 livres de fer et 73 poêles, soit une valeur de 4,692 dollars 11/100; il restait encore à vendre dans les magasins des Forges et dans celui des Trois-Rivières 101 poêles et 225,194 livres de fer'.

$\mathrm{Au}$ retour de Burton, en mars 1763, Haldimand pouvait présenter des comptes encourageants: les Forges avaient produit plus qu'en 1761 et rapporté davantage, et surtout il avait acquitté toutes

4. AC, B 21-1: 121. Plusieurs rapports (B 21-1: 108; B 21-2: 63 et d'autres) donnent 86,487, mais, dans une vérification du 28 septembre 1764, Courval fait remarquer que c'est bien 82,437 (B 21-2: 10); il faut tenir compte de cette correction dans les comptes ultérieurs d'Haldimand.

5. Les 180 poêles des comptes de 1762 sont des poêles fabriqués en 1761 . Il n'a pas été fait un seul poêle sous la double administration Haldimand.

6. AC, B 21-1: 108s; B 21-2: 3. Soit \$35,700. d'aujourd'hui.

7. Soit $\$ 18,400$. d'aujourd'hui.

8. AC, B 21-2: 63. Il le paya à raison de 60 dollars le millier: 2,220 dollars, soit $\$ 13,320$. d'aujourd'hui.

9. AC, B 21-1: 118, 124. Cependant, il restait dâ 21 poêles et 9,903 livres de fer. Sur les 180 poêles fabriqués, notons qu'il y en a un certain nombre qu'on réserve d̀ l'usage des Forges et des ouvriers; c'est ce qui explique qu'après la vente de 73 poêles il n'en reste que 101 dans les magasins. 
les dépenses du Gouvernement des Trois-Rivières avec l'argent qu'il avait encaissé par la vente du fer ${ }^{10}$. Amherst, pour sa part, se déclarait enchanté, écrivant qu'il valait bien la peine, non seulement de réparer les Forges, mais même de les agrandir et d'en augmenter la production ${ }^{11}$.

Lorsque Burton reprend la direction de son Gouvernement, le Canada appartient officiellement à l'Angleterre depuis près d'un mois, mais la signature du traité de Paris ne change rien pour le moment à l'administration du pays. En effet, comme ce traité accorde aux Canadiens dix-huit mois pour choisir entre le Canada et la France ${ }^{12}$ l'instauration du régime civil se trouve remise au 10 aout 1764 et, pendant cet intervalle, les Forges, comme le reste du pays, continuent d'être contrôlées par l'armée et de fonctionner. Les comptes des Forges, du mois de mars au 25 octobre (mais il n'est. pas sûr que les Forges aient repris leur activité avant le mois de mai), nous apprennent qu'on a produit 99,520 livres de fer ${ }^{13}$; de plus, on recommence à produire des poêles, mais, cette fois, comme le Canada est devenu définitivement une colonie anglaise, on laisse de côté l'ancien modèle français avec fleurs de lis et l'on fabrique un "nouveau modèle avec les armes d'Angleterre": 50 gros poêles à 21 dollars $2 / 3$ chacun et 50 petits à 20 dollars, outre 90 plaques de fer pour poêles de briques ${ }^{14}$. Mais Burton ne présida pas plus longtemps à l'industrie des Forges: le 25 octobre, il quitte de nouveau son Gouvernement des Trois-Rivières pour aller remplacer à Montréal le général Gage qui venait d'être promu à New-York ${ }^{15}$, et c'est encore Haldimand qui revient gouverner aux Trois-Rivières. Pour les Forges, la période de travail tirait à sa fin: elles fonctionnèrent encore pendant près d'un mois et produisirent, pendant ce temps, 37,462 livres de fer ${ }^{16}$. L'année 1763 s'avérait donc encore fructueuse:

10. De mai à décembre 1762, les dépenses du Gouvernement et du Secrétariat se chiffraient à 1384 dollars 30/100, soit \$8,305. d'aujourd'hui (AC, B 21-1: 43-46). Pour défrayer toute l'administration Haldimand (mai 1762-mars 1763), on prit 45,000 livres de fer sur les 150,476 fabriquées (AC, B 21-2: 15).

11. Amherst à Haldimand, le 25 janvier 1763, BM 21661, 262v.

12. Doc. const., 86: art. 4 du traité.

13. AC, B 21-2: 16 .

14. AC, B 21-2: 13. Les anciens poêles français se vendaient 18 dollars $1 / 3$.

15. Le général Gage était rappelé à New-York pour y remplacer le génóral Amherst qui rentrait en Angleterre.

16. AC, B 21-2: 25, 78. D'après les comptes, les Forges auraient fonctionné jusqu'au dernier jour de novembre. 
136,982 livres, 100 poêles et 90 plaques. La production n'était en rien inférieure à celle de l'année précédente, et comme il ne s'était présenté aucune dépense extraordinaire en 1763, la valeur approximative de 10,300 dollars que l'on avait fabriquée en fer couvrait facilement les frais d'exploitation et les dépenses du Gouvernement ${ }^{17}$. Les Forges cependant ne sont toujours pas rétablies à neuf: Haldimand ne perd pas de vue le projet de les refaire et il prend même la peine de recommander à Amherst un spécialiste suédois, de passage aux Trois-Rivières, et qu'il déclare très habile dans l'industrie du fer $^{18}$.

Le changement d'administration qui s'annonçait pour le mois d'aout 1764, ne permettait pas à l'armée de rien entreprendre d'envergure; aussi, quand le travail des Forges reprend au début de juin de cette même année, on ne s'applique qu'à fondre de vieilles pièces d'artillerie ${ }^{19}$. Un incident vint quelque peu bouleverser les comptes du méticuleux Haldimand. En juillet 1764, "ayant été obligé de faire netoyér le Magasin des Provisions pour detruire les Rats"20, Haldimand en profita pour faire peser le fer qui se trouvait dans ce magasin des Trois-Rivières ${ }^{21}$ : il en manquait plus de sept mille livres et 43 barres. Il supposa tout de suite que l'erreur pouvait venir de la différence des poids des Forges qui "sont de 11 à 12 livres par Cent plus legers, que Ceux du Magasin; ce qui seroit Cause, qu'au lieu d'avoir vendu le fer 36 fr le Cent, On l'auroit réellement donné meilleur Marché sans le Savoir" ${ }^{22}$. Il ouvrit une enquête: on constata que les poids du magasin des Trois-Rivières excédaient ceux des Forges de $7 \%{ }^{23}$, mais comment expliquer la disparition des 43 barres? Ou bien on s'était trompé dans les comptes ou il y avait eu vol; en ce dernier cas, écrit Burton, "comme les clefs des magasins étaient confiées à des sergents qu'on a changés souvent, il est difficile de dire qui est coupable" ${ }^{24}$. Des témoins

17. On n'a pas l'état des dépenses pour cette période; on sait seulement qu'il n'y eut pas de réparations extraordinaires.

18. Haldimand à Amherst, le 27 octobre 1763, BM 21662, 90. Ce Suédois avait nom Nordberg.

19. AC, B 21-2: 43, 50, 78 .

20. Haldimand à Burton, le 17 juillet $1764, \mathrm{BM} 21669,85 \mathrm{~s}$.

21. On le désignait sous le nom de "long Magasin du Roy", AC, B 21-2:26.

22. 36 francs le cent, c'est toujours 60 dollars le millier (on disait franc ou livre). BM 21669, loc. cit.

23. Haldimand à Burton, le 8 aout 1764, BM 21669, 92.

24. Burton à Haldimand, le 8 août 1764, BM 21669, 94. 
vinrent déposer à l'enquête que des soldats étaient allés, de nuit, chez divers forgerons pour offrir du fer en refusant de se faire connaitre ${ }^{25}$. Finalement, on découvrit le voleur: c'etait un soldat du 27ième Régiment qui, maréchal de son métier, s'était fait une fausse clef du magasin: "prenant son temps, lorsqu'il étoit lui même Sentinelle il a été decouvert par un baril de Beurre, quil vouloit vendre, \& il est Aujourd'huy, en prison" ${ }^{26}$. Cet incident, qui nous rappelle que le marché noir est de tous les temps, servit au moins a faire constater la différence des poids et à éviter de vendre à perte plus longtemps.

La production de 1764, par suite de l'expiration du régime mili taire, se trouve donc peu considérable: en quatre mois (les seuls mois d'opération de l'année), les Forges donnent 67,659 livres de $\mathrm{fer}^{27}$. C'était à peine la moitié du produit de 1763 . Bien que le produit de 1764 se trouvât réduit de moitié, il y avait encore surplus: il en avait couté 3,335 dollars $^{28}$ pour fabriquer du fer évalué à 4,059 dollars 54/100; il restait donc quelques centaines de dollars pour couvrir une partie des dépenses du Gouvernement ${ }^{29}$. Encore ici, le fer ne trouve pas immédiatement acheteur: de décembre 1763 à fin de septembre 1764, Haldimand en vend tout de même 17,624 livres pour environ 1,000 dollars et 33 poêles $^{30}$. Théoriquement, il valait toujours la peine de soutenir les Forges: "Il y à Actuellemt. dans le Magasin du Roy, écrit Haldimand en septembre, passé 370. Milliers Livres de fer en Barre, Sans Contér nombre de Poilles dont le produit est plus que Suffisant, pour retablir les forges à Neuf, et les faire Marchér Une anné Sans que le Public Soit obligé de faire Aucune Avance; et Si Elles Sont bien Ménagées, je Crois quelles pourroint payer par la Suitte les dépences de Ce Gouvernement" ${ }^{31}$.

25. AC, B 21-2: 27 (rapport sur la pesée du fer).

26. Haldimand à Burton, le 14 août 1764, BM 21669, 96.

27. AC, B 21-2: 43. D'après un mémorandum d'Haldimand, le travail a dur6 jusqu'au 30 septembre, ibid., 111.

28. AC, B 21-2: 21, 32-34, 52-56.

29. Encore à l'automne de 1764, Haldimand évalue le fer à 60 dollars le millier (Haldimand a Gage, le 25 novembre 1764, BM 21662, 176v.) Du 25 octobre 1763 au 24 octobre 1764, Gugy, secrétaire du gouverneur, fit au Bureau du Secrétaire pour plus de 400 dollars de déboursés à même les revenus de la vente du fer (AC, B 21-2: 72s).

30. AC, B 21-2: 53s.

31. Haldimand à Amherst, le 6 septembre 1764, BM 21661, 282. 
Mais au moment où Haldimand propose encore de rétablir les Forges, le régime militaire avait officiellement fait place au régime civil depuis près d'un mois ${ }^{32}$ : il était grand temps de remettre l'administration des Forges à l'autorité civile. Déjà, le 2 février, le général Gage écrivait de New-York que les Forges ne relevaient plus de son commandement ${ }^{33}$, mais ce n'est que le 17 septembre qu'il donne formellement instruction à Haldimand de régler les comptes des Forges et de tout remettre au pouvoir civil ${ }^{34}$. Haldimand, qui avait pris tant à cœur l'exploitation des Forges, avait lieu maintenant de s'inquiéter de ce qu'il en adviendrait; il regrettait de s'être bien dérangé pour peut-être donner à des particuliers le profit qu'il avait procuré au roi ${ }^{35}$. De fait, son rôle avait été très bien tenu: quand on songe qu'Haldimand, qui n'était que gouverneur intérimaire, a fait produire autant de fer que Burton, et cela dans une période de temps plus courte ${ }^{36}$, on doit reconnaître qu'Haldimand avait vu soigneusement à l'intérêt du roi et de l'armée. Et lorsque Haldimand dit qu'il s'est bien dérangé, ce n'est pas un vain mot: en attendant que les clients achètent et paient le fer, comme aussi en attendant que le payeur de l'armée veuille bien lui envoyer de l'argent de Montréal, Haldimand était obligé de fournir de l'argent personnel pour payer les ouvriers, et surtout d'en emprunter ${ }^{37}$.

Cramahé arriva aux Trois-Rivières, le 28 septembre, pour prendre charge officiellement de l'administration civile: il était nommé et envoyé par Murray. Ce dernier, dans l'article 55 des instructions qu'il avait reçues de Londres, n'avait été avisé ni de poursuivre l'exploitation du fer ni de la concéder à bail: on lui enjoignait seulement de voir à la préservation du domaine royal ${ }^{38}$. Aussi Cramahé se

32. Soit depuis le 10 août.

33. Gage à Haldimand, le 2 février 1764. BM 21662, 103.

34. Le même au même, le 17 septembre 1764 , ibid., 169.

35. Haldimand à Burton, le 8 aout 1764, BM 21669, 92; Haldimand à Gage, le 18 aout 1764, BM 21662, 173.

36. Burton avait gouverné pendant 27 mois, et Haldimand pendant 21.

37. Haldimand à Gage, le 27 décembre 1763, BM 21662, 95; ibid., 102: le même au même, le 25 janvier 1764 .

38. L'article 55 disait: “Attendu qu'il est démontré par les représentations de Notre gouverneur de Trois-Rivières que les forges du Saint-Maurice situées dans ce district, sont d'une grande importance pour Notre service: c'est par conséquent Notre bon plaisir qu'il ne soit concédé à aucun particulier aucune partie des terres qui ont servi à l'exploitation desdites forges et à la production du minérai ou qu'il semblera avantageux et nécessaire d'attacher a cet établissement soit pour avoir libre accès au fleuve Saint-Laurent soit pour en retirer le bois, le grain et le foin né- 
contenta-t-il de se faire délivrer un inventaire des Forges, de conseiller à Haldimand de vendre ce qui restait de fer et de repartir pour Québec au bout de six jours, emportant avec lui les registres et papiers civils du district ${ }^{39}$. Selon cet inventaire que d'autres documents nous aident à compléter, il restait dans le magasin des Trois-Rivières, 386,303 livres de fer et 148 poêles $^{40}$; le magasin des Forges n'en contenait plus que 12,564 livres ${ }^{41}$. Les réserves de l'industrie n'étaient guère plus alléchantes que celles de septembre 1760: 400 barriques de mine, de la fonte pour faire 6,000 livres de fer, 40 canons et autres pièces pouvant produire 64,000 livres $^{42}$. D'après Courval, il y avait encore 43 employés, dont un fondeur, deux martelleurs ${ }^{43}$, deux chauffeurs et deux gardes ${ }^{44}$. L'inventaire ajoute que "tous les ouvriers Et manœuvres qui ont Eté Employés aux forges de St Maurice sont payés En plain jusqu'au premier" octobre $^{45}$.

Haldimand, qui demeurait toujours sur les lieux à titre de commandant militaire ${ }^{46}$, se mit donc à vendre le plus possible de fer et de poêles, pour se conformer aux directives du commandant civil, Cramahé. Malheureusement, les conditions semblaient ne plus être aussi avantageuses. Jusque là, Haldimand avait pu vendre son fer à 6

cessaires ou pour servir de pâturage au bétail; en outre, qu'il Nous soit réservé en sus des terres requises pour les besoins susdits, un territoire aussi étendu que possible contigu auxdites forges ou à proximité de celles-ci, dont il sera disposé de la manière que Nous indiquerons et prescrirons ci-après." Doc. const., 173.

39. Haldimand à Gage, le 25 novembre 1764, BM 21662, 177. Haldimand ne comprit rien au voyage rapide de Cramahé: c'est qu'on avait décidé à ce moment-là de supprimer le Gouvernement des Trois-Rivières.

40. AC, B 21-2: 39s. Soit 79 poêles français et 69 poêles anglais.

41. AC, B 21-2: 51 (inventaire du 28 septembre 1764).

42. AC, B 21-2: 134 (inventaire remis à Cramahé, le 4 octobre 1764).

43. Courval dit que l'un commence à perdre la vue et que l'autre veut passer en France. Il s'agit en ce dernier cas, de Jean-Nicolas Robichon, arrivé au Canada en 1740; on peut facilement l'identifier, dans une lettre de Haldimand a Murray (BM 21666, 186: le 14 septembre 1764) où il est question de son fils encore gougeat. Ce Jean-Nicolas Robichon, selon Sulte, avait voulu rentrer en France en 1761, 1762 et 1763 (Sulte, Les Forges, 139s.); Sulte ajoute qu'il est parti pendant l'été de 1764: ce qui est presque exact, puisque Haldimand écrit à Murray, le 14 septembre. pour le recommander comme étant le meilleur ouvrier des Forges et très difficile à remplacer (BM 21666: loc. cit.).

44. Les membres du personnel permanent sont encore les mêmes: l'inspecteur Courval, le contremaître Voligny, Delorme, Imbleau, "Bellu", Robichon père et fils, Marchand père et fils, Terreau et Michelin (AC, B 21-2: 43, 56).

45. AC, B 21-2: 134 (inventaire remis à Cramahé, le 4 octobre 1764).

46. Gage a Haldimand, le 26 octobre 1764, BM 21662. 
dollars le cent, mais le fer d'Europe faisait déjà concurrence à celui des Forges et d'autant plus facilement que le fer d'Europe tenait lieu de lest pour les navires qui traversaient en Amérique; on lui offrait même d'en acheter à 4 dollars le cent; de plus, Haldimand avait oublié d'ajouter 12 livres de fer par cent "pour le Vendre Marchandable, Ce qui fait une grande diminution"47. Quant au prix des poêles, il ne semble pas avoir subi de dépréciation ${ }^{48}$. Malgré tout, Haldimand trouva des acheteurs à un prix convenable: les comptes de cette période indiquent que le fer a été vendu tantôt à 6 dollars le cent, tantôt à 5 dollars. Certains acheteurs se présentèrent pour une quantité considérable: Jacques Terroux, marchand de Québec, en acheta pour une valeur de 14,000 dollars ${ }^{49}$; Tonnancour, des Trois-Rivières, y alla de 15,786 livres et de 22 poêles $^{50}$; de septembre 1764 à mars 1765, Malcom Fraser, officier militaire des TroisRivières, avait vendu, de son côté, 120,000 livres et 55 poêles ${ }^{51}$. Toutes ces ventes et quelques autres servirent à payer les dépenses contingentes du Gouvernement ${ }^{\text {b2 }}$.

Finalement, comme Haldimand se préparait à quitter TroisRivières pour New-York, il fallut en venir à un règlement définitif des comptes. Le 5 juin 1765, Gage donnait instructions à Haldimand de dresser un état de comptes pour la période comprise entre la main mise de l'armée sur les Forges et leur remise au pouvoir civil; s'il restait du fer ou des poêles, il fallait les remettre à Burton qui

47. Haldimand à Gage, le 25 novembre $1764, \mathrm{BN} 21662,176 \mathrm{v}$.

48. AC, B 21-2: 99. Sept poêles ont couté 130 dollars, soit environ 18 dollars chacun (ou $\$ 108$. d'aujourd'hui).

49. AC, B 21-2: 70 (compte du 29 mai 1765, compte qui servit a acquitter un égal montant de dépenses).

50. AC, B 21-2: 85. Il est intéressant de noter comment Tonnancour s'y prit pour acquitter le compte de 6467 livres 18 sols:

"Payement que J'ay fait:

388 Ecus de 6 livres 12 s......................... 2560.16

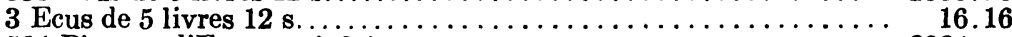

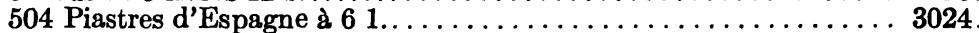

18 Portuguaises à $481 \ldots \ldots \ldots \ldots \ldots \ldots \ldots \ldots \ldots \ldots \ldots \ldots, 864$.

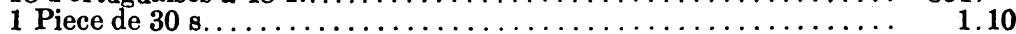

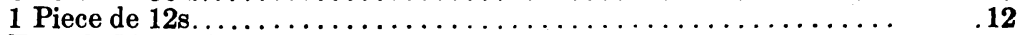

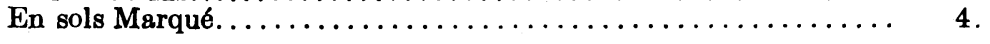

51. AC, B 21-2: 68s.

6467.18

52. AC, B 21-1: 76 . 
les vendrait et en verserait le profit dans la caisse de l'armée ${ }^{63}$. De son côté, Burton ordonnait à Haldimand de tout remettre maintenant à l'administration civile ${ }^{54}$. Haldimand profita d'un séjour à Québec pour confier tout ce qui restait à François Lévesque, marchand de Québec, qui avait ordre de tenir tout le dépôt à la disposition de Burton ${ }^{55}$. Par un reçu du 5 juillet 1765, "F. Levesque \& Co." reconnaît avoir reçu ainsi en dépôt 310,000 livres de fer et 87 poêles $^{56}$.

L'administration des Forges par l'armée anglaise était donc bel et bien terminée: qu'allaient désormais devenir les Forges? Depuis que le Canada appartenait définitivement à l'Angleterre, il s'était présenté des particuliers pour en demander l'exploitation à bail. John Marteilhe, marchand de Québec, fut le premier, semble-t-il, à réclamer le bail des Forges. Dans un mémoire qu'il adresse au Board of Trade et qui fut lu à Londres le 21 juin 1764, il demande que s'il est décidé que les Forges seront données à bail, on lui accorde la préférence, et il a soin de joindre à son mémoire une description des Forges et de leur production ${ }^{57}$. En novembre 1764, Haldimand écrit a Cage que "le petit M. Kenzée, l'associé de Ocks, a passé icy Un Mois \& y a fait un vollume d'observations, Avec lesquelles il est passé a Londres tres bien recomande'"58. L'exploitation a bail avait cependant des adversaires. En septembre de la même année, Courval avait présenté un mémoire pour demander que l'exploitation soit poursuivie au nom du roi: la qualité du fer, écrit-il, ne convient qu'aux vaisseaux et à l'artillerie du roi; l'exploitation ne peut être bien considérable, parce que le bois et la mine ne sont pas très abondants et, si l'on veut assurer des revenus durables au Gouvernement des Trois-Rivières, il faut se contenter de produire 200 ou 300 milliers de fer par année: or, des particuliers, pour faire immédiatement de gros profits, ruineraient le tout en cinq ans; enfin, il faut l'autorité pour appeler aux Forges les hommes nécessaires et les tenir a l'ouvrage ${ }^{59}$. Vers le même temps, le contremaître Voligny présenta

53. Gage à Haldimand, le 5 juin 1765, BM 21662, 199.

54. Burton à Haldimand, le 21 juin 1765, BM 21669, 135.

55. Haldimand a Burton, le 5 juillet 1765, ibid., 138.

56. AC, B 21-2: 110 .

57. C.O. 42 , I-1: $159-165$.

58. Haldimand a Gage, le 25 novembre 1764, BM 21662, 176v.

59. AC, B 21-2: 41-42v. (mémoire du 20 septembre 1764). 
lui aussi un mémoire pour demander que le roi continue de se charger de l'exploitation: comme le fait Courval, il explique qu'une compagnie privée ruinerait les Forges d'un seul coup; Voligny fait encore remarquer que les Forges sont nécessaires à la région et qu'elles ont eu, entre autres heureuses conséquences, d'amener les paroisses avoisinantes à se livrer à la culture du sol avec plus d'assiduité afin de nourrir la population ouvrière ${ }^{60}$.

Malgré ces divers mémoires, le gouvernement anglais ne prit aucune décision sur l'exploitation des Forges, ni pour la poursuivre par régie d'État ni pour la concéder à bail ${ }^{61}$. Au printemps de 1765 , on renvoie les ouvriers sans les payer et quelques-uns passent en France; en octobre, on congédie Courval sans le rembourser d'une partie de ses gages ni des sommes qu'il a avancées ${ }^{62}$ et l'on poste sur les lieux une douzaine de militaires pour surveiller les biens de l'État ${ }^{63}$.

La production du régime militaire reste bien inférieure à celle du régime français, elle n'en atteint même pas la moitié de la production moyenne $e^{64}$. Elle demeure tout de même impressionnante, quand on se rappelle qu'il ne s'agit toujours que d'une exploitation temporaire, entretenue pour tenter de réveiller l'économie d'une région qui, à l'instar de ses voisines, a subi le dur contre-coup de la guerre de Sept ans. D'octobre 1760 à octobre 1764, on fabrique 482,901 livres de fer et 280 poêles, soit une valeur de plus de 25,000 dollars $(\$ 150,000$. d'aujourd'hui). Cette production amène Burton et Haldimand à

60. AC, B 21-2: 64-66. Ce mémoire n'est pas signé, mais l'auteur écrit qu'il a consulté son père, directeur des Forges pendant 13 ans: ce ne peut être, par conséquent, que Voligny, fils de l'ancien directeur Hertel de Rouville.

61. Ce n'est qu'en 1767 que le roi concédera les Forges à Christophe Pélissier et Cie, pour une durée de seize ans (AC, B 21-2: 122-128).

62. AC, B 68: 38 (Gugy à Haldimand, le 18 novembre 1765). Gugy ne put luimême retirer l'argent qu'il avait prêté à Courval. Selon Gugy, Murray aurait refusé de payer les ouvriers, en soutenant que c'était à Haldimand de le faire.

63. Ibid.

64. John Marteilhe, dans un mémoire de 1764, se basant sur la production de 1756 qui lui semble le plein rendement des Forges en un an, donne une valeur de 141,432 livres 13 sols; comme le cent de fer se vendait alors environ 30 livres, selon Franquet, on obtient le poids approximatif de 400,000 livres. A comparer avec la production du régime militaire:

1761: 127,784

1762: 150,476

1763: 136,982

1764: 67,659

(C.0. 42, I-1: 159). 
dépenser, dans la région, plus de 20,000 dollars (soit $\$ 120,000$.): de cette somme, au moins le tiers ${ }^{65}$ fut distribué en salaires réguliers aux treize membres du personnel des Forges; une autre partie, qui dut être considérable, fut versée aux nombreux journaliers que l'on embauchait suivant les saisons, et aux habitants des environs qui étaient en mesure de fournir des provisions et du fourrage. Haldimand écrit en $\mathbf{1 7 6 4}$ que les Forges "font Subsister nombre de familles \& fournissent presque le Seul Argent qui Circulle icy"66. Même si la prodigalité des ouvriers scandalise Haldimand et empếche que tout cet argent demeure dans le Gouvernement des Trois-Rivières ${ }^{67}$, les Forges n'en constituaient pas moins une manne très précieuse dans un pays qui ne pouvait plus commercer avec la France et qui ne pouvait le faire encore, régulièrement, avec l'Angleterre. La substitution de l'équipe de Courval par un peloton de militaires, dut créer chez la population des Forges le plus complet désarroi. De 72 âmes qu'elle était en 1760 , cette population était passée à $273^{68}$ et, même si elle commençait quelque peu à prendre figure agricole ${ }^{69}$, elle n'en vivait pas moins qu'en fonction de l'industrie du fer. En cela comme en d'autres points, elle subissait le bouleversement néfaste que l'instauration du nouveau régime civil imposait partout au pays.

65. On donnait, par mois, en salaires réguliers, 892 livres 12 sols; ce qui fait pour 48 mois, 42,844 livres ou 7,140 dollars.

66. Haldimand a Amherst, le 25 octobre 1764, BM 21661, 283: Voligny estime à peu près de 100,000 livres par année (ou $\$ 100,000$. de nos jours) l'argent que les Forges font circuler dans le Gouvernement (AC, B 21-2: 66).

67. Il écrit: "L'Argent ny Sejourne pas longtemps, apeine les Ouvriers ont ils reçu leurs Sallaires quils le portent Ches les Marchands, et Ceux Cy l'envoyent bientôt a Québec pour de nouvelles Marchandises; je Suis persuadé qu'au bout de l'année il ne reste pas 100 francs dans la Bource d'aucun particullier; ils Aiment en general à jouir, et pensent rarement Au Lendemain." (Lettre à Amherst, le 5 décembre 1762, BM 21661, 256).

68. RAPQ (1936-37), 119 (recensement pour l'année 1765).

69. Le recensement de 1765 donne 809 arpents ensemencés, 29 bœufs, 107 vaches, 67 taurailles, 115 moutons, 89 chevaux et 184 cochons. Le village, qui comprenait 55 maisons, avait été desservi par le récollet Amiot jusqu'en 1762; en 1763, le P. Augustin Quintal y fait "les fonctions Curialles", puis, en 1764, occasionnellement, le grand-vicaire Perrault: voir aux archives de l'Evếché des Trois-Rivières, les Registres des Trois-Rivières: Forges Saint-Maurice, 6, 1740-1762 (ces registres se poursuivent de fait jusqu'en 1764). 


\section{BIBLIOGRAPHIE}

\section{Sources manuscrites}

Ce sont les Papiers Haldimand que nous avons utilisés pour la majeure partie de cette étude. Les originaux (auxquels nous référons par le sigle BM) sont conservés au British Museum et constituent les volumes 21661 à 21892 des Additional Manuscripts. Nous en avons cité les vol. 21661, 21662, 21666 et 21669, dont l'inventaire a été publié dans le Rapport des Archives publiques du Canada, 1884, 1-25, 80-96, $109-116 ; 1885,137-147$. Les Archives du Canada ont la copie manuscrite de ces Papiers, classés à Ottawa sous le sigle B, de 1 à 232. Nous avons cité occasionnellement les volumes 7 et 68 , inventoriés dans le même Rapport, 1884, 102-105; 1886, 517-520. C'est surtout le volume 21 (divisé en deux parties: B 21-1, B 21-2) que nous avons exploité, parce qu'il contient presque uniquement des pièces relatives à l'administration des Forges: voir l'inventaire dans le même Rapport, 1885, 183-188.

Enfin, nous avons eu recours à quelques documents conservés au Colonial Office, sous le sigle C.O. 42, dont nous avons cité la première partie du volume I, inventorié dans le Rapport des Archives publiques, 1921, 1-8.

La Bibliothèque de l'Université Laval a fait reproduire sur microfilms, plusieurs volumes des Papiers Haldimand et des documents du Colonial Office.

\section{Sources imprimées}

Le Rapport des Archives publiques du Canada pour l'année 1918 a édité les proclamations du régime militaire, dans l'appendice $B$, qui couvre 197 pages.

Le Rapport de l'Archiviste de la Province de Québec de 1924-25 a publié, p. 201228, les protestations des Chambres de Commerce de France contre le projet de céder le Canada à l'Angleterre: ces protestations démontrent que les marchands français considéraient toujours le Canada comme une colonie profitable; on ne parle cependant pas du fer canadien.

Dans le Rapport du même archiviste, pour 1936-37, on trouvera le recensement de 1765, fait dans les Gouvernements de Montréal et des Trois-Rivières.

Etudes

L'exploitation des Forges, sous le régime militaire, avait été jusqu'ici ou ignorée ou traitée d'une façon sommaire.

L'abbé N. Caron, Deux yoyages sur le Saint-Maurice (Trois-Rivières, P.-V. Ayotte, s.d.), VI-322 pp. L'auteur consacre à la question quatre courts paragraphes (p. 260s.), dont un seul porte directement sur l'exploitation. Il note, avec justesse, que "pendant tout le règne militaire qui dura 4 ans, les ouvriers pouvaient encore se croire au temps de la domination française".

Benjamin Sulte, Les Forges Saint-Maurice (Montréal, G. Ducharme, 1920), 216 pp. III. Vol. VI des Mélanges historiques. Son chapitre sur le régime militaire couvre les pages 129-141; on aurait aimé que l'auteur parlât moing des feux des Trois-Rivières et des mariages, baptêmes et sépultures des Forges, et beaucoup plus de l'exploitation. Des six ou sept pages qu'il consacre aux Forges, il y en a quatre pour résumer l'inventaire de septembre 1760.

Benoit Brouillette, Le developpement industriel de la vallee du St-Maurice (Les TroisRivières, Les Éditions du Bien Public, 1932), 54 pp. No 2, série A des Pages 
trifluviennes. Il écrit, page 12: "Après la conquête, les forges furent remises au gouvernement militaire qui n'en put rien tirer; elles demeurèrent inactives jusqu'en 1767." Comme Sulte, dans le chapitre qu'on vient de citer, ne dit pas que les Forges ont produit ou rapporté quelque chose, on peut voir d'où vient l'erreur.

R.C. Rowe, The St. Maurice Forges, dans The Canadian Geographical Journal, IX (1934), pp. 14-22. Ill. Consacre quelques lignes au régime militaire; il écrit que le fer, entre autres produits, a influencé la décision de l'Angleterre.

Albert Tessier, Aux sources de l'industrie américaine: les Vieilles Forges. No 3 du vol. I de la collection Les Cahiers Reflets (janvier 1945), 35 pp. Ill. Les pages 24-26 sont consacrées au régime militaire.

Albert Tessier, Les Anglais prennent les Forges au sérieux, dans le Cahier des Dix, XIV (1949), pp. 165-185. Les pages 165-170 donnent un bon résumé de la période. L'auteur, qui avait déjà abordé le problème en 1945 , fut le premier à démontrer que les Forges avaient connu, sous le régime militaire, une grande activité.

\author{
Marcel TRUDEL, \\ professeur d'histoire du Canada d \\ l'Université Laval.
}

\title{
COURS DE L'INSTITUT D'HISTOIRE À L'UNIVERSITÉ DE MONTRÉAL
}

Nos lecteurs sont priés de se reporter à notre Chronique de l'Institut. Ils y verront l'annonce des cours de notre Institut pour cette année 1951. Professeur: M. Guy Frégault. Sujet général des cours: Pierre de Rigaud de Vaudreuil Cavagnal en Louisiane.

Sujets de chacun de ces cours:

16 octobre: La Louisiane

17 octobre: Politique indigène

23 octobre: La révolte des Chactas

24 octobre: Histoire administrative

Nous comptons sur la fidèle assiduité de nos lecteurs à ces cours de notre Institut. 